\title{
EFFECTIVE RADIANT FLUX FOR NON-IMAGE FORMING EFFECTS - IS THE ILLUMINANCE AND THE MELANOPIC IRRADIANCE AT THE EYE REALLY THE RIGHT MEASURE?*
}

\author{
Kai Broszio, Martine Knoop, Mathias Niedling, and Stephan Völker
}

\author{
Lighting Technology Technische Universität Berlin, Germany \\ kai.broszio@tu-berlin.de
}

\begin{abstract}
Research indicates that intrinsically photosensitive Retinal Ganglion Cells are not evenly distributed or evenly sensitive throughout the retina. Still, most research looking into non-image forming (NIF) effects uses an integral measured quantity, illuminance or melanopic weighted irradiance, to represent the amount of light at the participants' eye level. This paper describes a theoretical approach to define the effective radiant flux for stimulating the ipRGCs, taking into account a spatially resolved sensitivity. Research on retinal sensitivity is scares and not yet substantial, but the methodology can easily be adopted when areas of specific sensitivity are set. Preliminary results indicate that, with similar vertical illuminances and spectral power distribution, typical office lighting solutions might have a lower NIF effectiveness than settings with higher luminances in the central part of the field of view. This could explain why research on NIF effects is inconclusive, even though reported lighting conditions are similar.
\end{abstract}

Keywords: inferior light exposure, nasal light exposure, NIF effects, office lighting, retinal sensitivity

\section{INTRODUCTION}

Since the discovery of the intrinsically photosensitive Retinal Ganglion Cells (ipRGCs) in 2002, non-visual effects or non-image forming (NIF) effects of light have become increasingly important in lighting research, development and design of lighting solutions. In this, lighting is, for example, used to increase alertness or sleep quality, to reduce desynchronization of the circadian rhythm or to treat seasonal affective disorders. The majority of the research looks into dependencies of amount, spectral power distribution, length and temporal distribution of light stimuli. Not in focus but also of interest seems to be the dependency on directionality and position of the light source, as only a small number (less than $1 \%$ ) of the retinal Ganglion Cells are photosensitive [1], and they are not evenly distributed throughout the retina (review in [2]).

Only a few publications on the impact of spatial light distribution include the description of lighting conditions in the experimental set-up. The majority of these studies were conducted between 1992 and 2005 (review in [3]). The offered lighting conditions differed greatly from one study to another. They ranged from 5 to 1000 lx, vertical illuminance at eye level, with varying colour temperature from warm white to cool white, realized by polychromatic light sources; fluorescent lamps, halogen lamps or LEDs. Partial retinal exposure was realised by using light boxes at defined positions in the field of view, or applying modified eye shields on subjects looking into a uniformly lit half dome. All studies took place at night time, sometime between 22:00 and 3:30. The lighting conditions were offered for

\footnotetext{
* On basis of report at the European conference LUX EUROPA 2017, Ljubljana, Slovenia, 18-20 September
} 


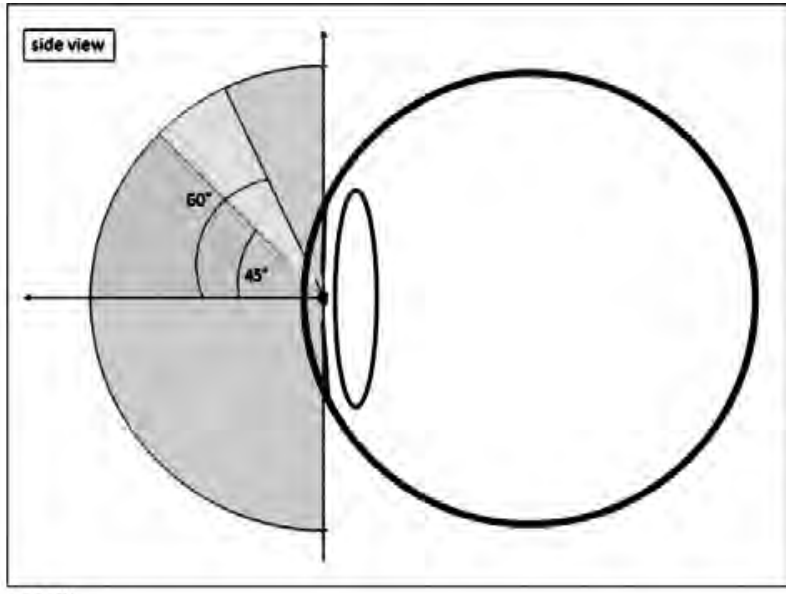

[F13]

Fig.1. Approximation of spatial sensitivity due to ipRGC density or sensitivity as found in a small number of studies based on [16] with areas of "little" (yellow) and "good" (green) response to induce NIF effects

60-240 minutes and melatonin suppression was used to study the impact of light source size and / or position. The number of participants per study ranged from 6 to 32, with varying age.

The studies suggest that large sources are more effective than small sources [4]. Binocular light exposure realises a higher melatonin suppression than monocular light exposure (spatial summation in [5, 6]). Additionally to that, the studies indicate that, in human beings, nasal exposure is more effective than temporal exposure $[7,8]$. Inferior retinal light exposure seems to induce a greater response than superior exposure $[9,11]$. Piazena et al. partly confirmed these findings [12]. Superior warm white light exposure $(8001 \mathrm{x}, 2666 \mathrm{~K})$ resulted in the reduced and delayed melatonin suppression in comparison to inferior exposure realising the same lighting level at the eye. However, similar cool white light levels $(6060 \mathrm{~K})$ caused comparable NIF responses for both inferior and superior light exposure.

Considering the above mentioned, it is questionable if illuminance levels or melanopic irradiance levels, currently referred to in studies with respect to NIF effects of light, are adequate parameters in terms of comparability, being integrally measured values of the full visual field. Retinal illuminance would respect human anatomic restrictions [13], and can be measured with adjusted illuminance sensors (e.g. $[14,15])$. Nonetheless, a more distinct subdivision within the human field of view might be required. The number of studies is too small to define areas with different ipRGC sensitivities. Despite this, the suggestion for approximation areas of "no", "little" and "good" effectiveness is given by FGL (Fördergemeinschaft Gutes Licht, 2014), Fig. 1, [16].

The aim of the presented study is to look into the range of the spatially resolved, effective radiant flux stimulating the ipRGCs for different distribution of the incident light under a constant (full visual field) illuminance level at the eye.

\section{METHODOLOGY}

The study described in this paper uses a theoretical approach to evaluate the impact of higher sensitivity for nasal and inferior light exposure. For this, a methodology to evaluate the spatially resolved illuminance contribution of defined areas in the field of view to the overall vertical illuminance at the eye was defined. Luminance images are converted into matrices containing luminance values for each pixel. Using another matrix that holds the opening angle $\omega_{s}$ and an additional matrix with the tilt angle $\vartheta$ of each pixel, it is possible to calculate the illuminance contributions. Generally, the following equation applies:

$$
E_{p}=\int L(\vartheta, \varphi) \cos (\theta) d \omega_{s}
$$

where $E_{p}$ is the illuminance, $L$ is the luminance of each pixel, which position is determined by $\theta, \varphi, \omega_{s}$ solid angle, $\vartheta$ is the tilt angle.

The resulting matrix comprises the illuminance contribution of each pixel to the integral vertical illuminance. Hence it is possible to define regions of interest in the field of view and to calculate the illuminance at eye-level caused by each region.

\subsection{Test Room}

A complete LED backlit test room with typical cell-office dimensions $(5 \mathrm{~m}$ width, $4 \mathrm{~m}$ length and $2.8 \mathrm{~m}$ height) at the Chair of Lighting Technology of TU Berlin was used for this study. This test room is equipped with 1470 individually addressable LED panels with a size of $18 \times 18 \mathrm{~cm}$, covered by a diffusing material. Each panel holds 36 mid-power, cool white or warm white LEDs. The correlated colour temperature (CCT) of the LED panels behind the diffusing material was measured with a 'Specbos 1201' spectrometer by Jeti Technical Instruments. The cool white setting has approx- 


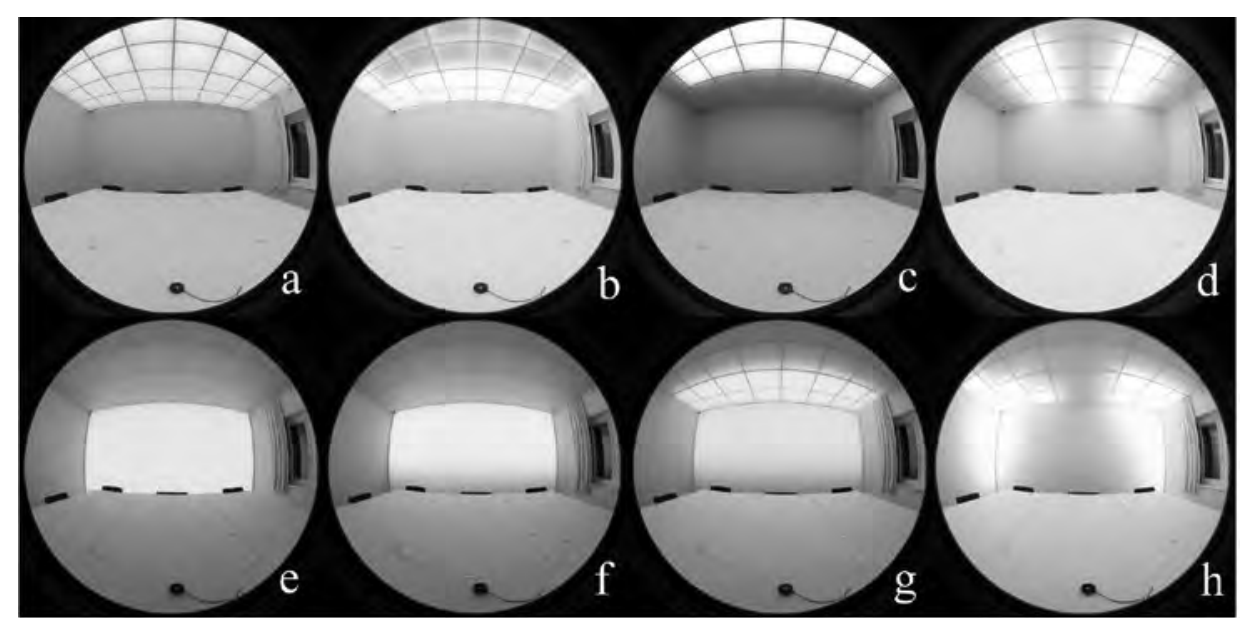

Fig.2. Lighting scenes (a - d typical office like; e - f non-typical like)

imately $5900 \mathrm{~K}$, the warm white setting $2800 \mathrm{~K}$. Mixed settings have 4400 to $4500 \mathrm{~K}$, depending on the used lighting scenes. The cool and warm white LED panels are arranged in a checkerboard pattern to ensure good uniformity and mixing characteristics if used together as well as separately. With this installation, it is possible to set specific luminance distributions for separate fields of the walls and ceiling with different CCTs. Twelve luminaires in the middle part of the ceiling are designed to optimise NIF effects; offering a range between 2000-20000 K.

\subsection{Settings}

Within this study, eight different luminance distributions, some very similar to typical electric lighting solutions for offices, some very different, were set to realize a constant vertical illuminance of $5001 \mathrm{x}(+/-2.5 \%)$ at eye level, Fig.2. This was

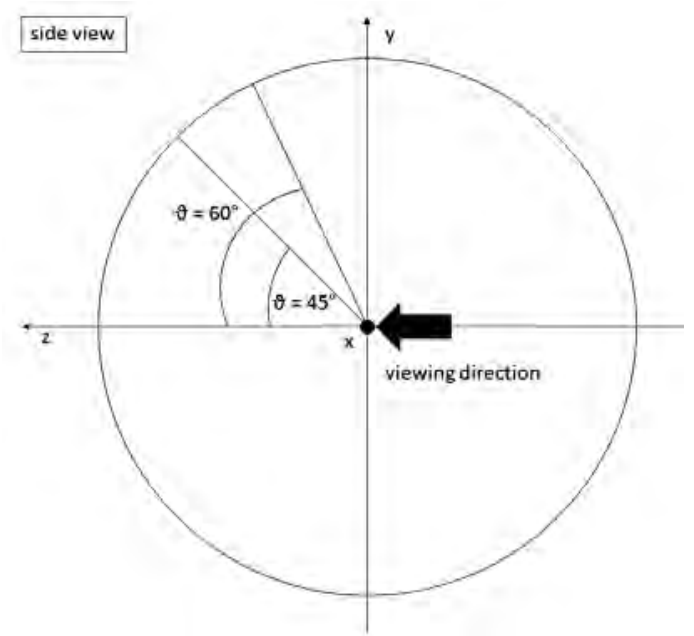

measured at $1.20 \mathrm{~m}$ above floor level by a luxmeter MX-ELEKTRONIK Mini-Lux with a $V(\lambda)$ - and cosine-corrected Si-photometer head. Additionally the horizontal illumination at $0.85 \mathrm{~m}$, at a fixed position on the desk was measured with a cosine-corrected luxmeter LMT Pocket-Lux 2. CCT of the lighting scenes ranged from $3900 \mathrm{~K}$ to $4900 \mathrm{~K}$.

\subsection{Measurement and Processing}

The variable in this study, the luminance distribution of the eight chosen scenes, was measured by a luminance camera 'LMK mobile advanced' based on a CANON EOS550D by TechnoTeam GmbH equipped with a $4.5 \mathrm{~mm}$ object lens (circular fisheye lens) with an angle of coverage of $140^{\circ}$. For each setting HDR luminance images were taken.

Based on the approximation of spatial sensitivity by FGL (Fördergemeinschaft Gutes Licht) (Fig. 1), and the anatomic restrictions (e.g. light shielded by

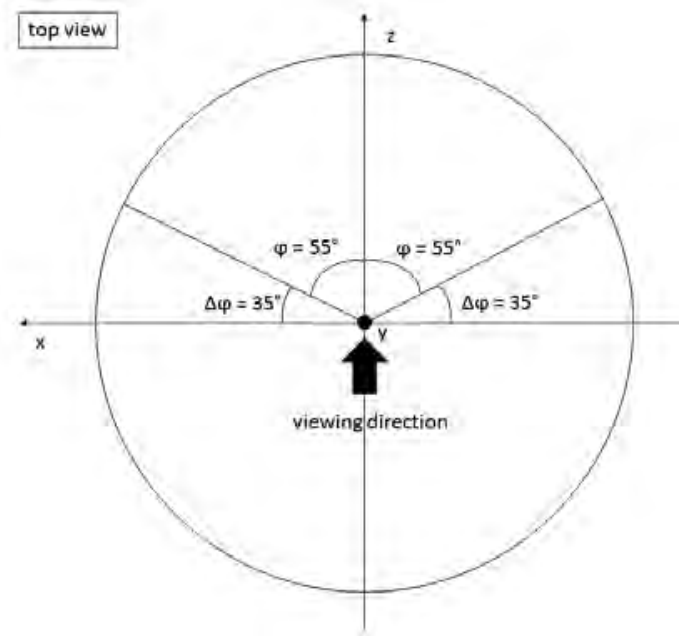

Fig. 3. Schematic of the angles in the visual field (based on [13] and [16]) 


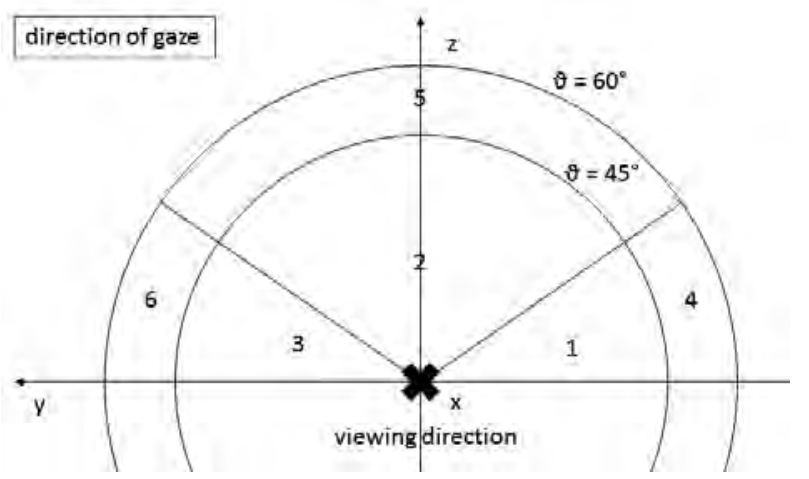

Fig.4. Overview of the region in the field of view

the nose), following areas of interest were chosen to demonstrate the potential and the consequences of a spatial differentiation (Fig. 3 and Fig. 4):

- $\vartheta=0^{\circ}$ to $45^{\circ}$ and $45^{\circ}$ to $60^{\circ}$

- $\varphi=0^{\circ}$ to $55^{\circ}$ and $0^{\circ}$ to $-55^{\circ}$ (to both sides of the direction of gaze).

Light coming from region 1 (Fig. 4) illuminates only the nasal part of the right eye's retina. Light in region 2 causes illumination of the lower part of both retinas. Light from region 3 illuminates the nasal part of the left eye. These angles are believed to have a good impact on NIF effects, while angles between $\vartheta=45^{\circ}$ to $60^{\circ}$ are supposed to have only weak effect and higher angles have no effect [16]. Moreover, region 2 is of special interest since the illumination of both retinas is found to cause the higher melatonin suppression $[5,6]$.

\section{RESULTS}

The methodology was applied to the eight lighting scenes shown in Fig. 2. For each region, the illuminance contribution was calculated. Table 1 holds this value represented as percentage of the measured vertical illuminance for better comparability. The lighting scenes with light mostly coming from the ceiling $(\mathrm{a}-\mathrm{d})$ show in general low values. The $\vartheta$ equal to $(0-45)^{\circ}$ region contributes only with 10 to $26 \%$ to the integral illuminance. If $\vartheta=0^{\circ}$ extended to $60^{\circ}$ this increases up to $44 \%$. On the other hand, lighting scenes $(\mathrm{e}-\mathrm{h})$, with light mostly coming from the opposite wall, have relatively high values. The $\vartheta=0^{\circ}$ to $45^{\circ}$ region contributes with $35-51 \%$, in the extended $\vartheta=0^{\circ}$ to $60^{\circ}$ region up to $58 \%$. In case region 2 gets a higher weighting, taking the findings of Wang [5] and Brainard [6] into account, the differences between lighting scenes $\mathrm{a}-\mathrm{d}$ and $\mathrm{e}-$ $\mathrm{h}$ become even larger.

\section{CONCLUSSION AND DISCUSSION}

A luminance camera based evaluation method to determine the spatially resolved partial illuminance values was developed. Here regions were chosen to fit illumination of the lower left and right eye's nasal part of the retina and the illumination of the lower halves of both retinas simultaneously. Typical and non-typical office lighting scenes were investigated. Data showed that the more standard-like office lighting scenes cause only weak illumination of the defined regions. Logically, lighting of the opposite wall leads to much higher contributions from these regions. With comparable spectral power distribution and vertical illuminances, typical, electric, lighting solutions for offices will result in a lower efficiency to induce NIF effects than settings with higher vertical luminances in the central part of the field of view, such as day lit rooms or lighting solutions with wall washers.

It needs to be pointed out, that there are only few studies indicating differences in melatonin suppression if superior or inferior halves of the retina are illuminated and even fewer showing differences in nasal and temporal parts of the retina. For now, an exact determination of regions and their respective sensitivity weighting cannot be made. Furthermore, these studies were executed at night time looking into the resulting suppression of melatonin levels. Rüger et al. already showed that suppression of melatonin does not per se lead to reduced sleepiness when only parts of the retina are illuminated [17]. Additionally to that, these results are not directly applicable for daytime responses. During daytime, the mode of action for NIF effects is still not well enough understood. Cones, with their incidents mostly in the centre of the visual field, are considered to influence NIF effects as well [18]. Resulting, it could be that, at least under daytime conditions, the distribution and spectral sensitivity of more than one receptor has to be taken into account.

In this respect, this method is a theoretical approach. Nonetheless, it is an aspect of interest, as it could explain why some studies do find NIF effects, and others do not, even though vertical illuminances and spectral power distribution of the light sources applied are similar. In order to compare these studies and to allow future adjustments to areas of interests and their specific sensitivities, it is proposed to look into the representation that accounts for the 
TABLE I. illuminance values

\begin{tabular}{|c|c|c|c|c|c|c|c|c|c|}
\hline \multirow[b]{2}{*}{ Regions } & & \multicolumn{8}{|c|}{ Lighting scenes } \\
\hline & & & & & & & & & \\
\hline & & $\mathrm{a}$ & $\mathrm{b}$ & c & d & e & $\mathrm{f}$ & g & $\mathrm{h}$ \\
\hline \multirow{2}{*}{$\begin{array}{l}\text { Illumi- } \\
\text { nance }\end{array}$} & $\mathrm{E}_{\mathrm{v}}[\mathrm{lx}]$ & 506 & 511 & 504 & 500 & 497 & 500 & 507 & 496 \\
\hline & $\mathrm{E}_{\mathrm{h}}[\mathrm{lx}]^{\mathrm{a}}$ & 657 & 694 & 619 & 640 & 263 & 276 & 329 & 338 \\
\hline & $E_{v}[\%]^{b}$ & 2.8 & 3.2 & 2.0 & 2.8 & 9.5 & 10.6 & 7.5 & 8.5 \\
\hline & $E_{v}[\%]^{b}$ & 15.1 & 19.2 & 5.6 & 17.3 & 24.3 & 29.2 & 28.6 & 19.1 \\
\hline & $E_{v}[\%]^{b}$ & 2.8 & 3.1 & 1.9 & 2.6 & 9.9 & 11.1 & 7.7 & 7.3 \\
\hline & $E_{v}[\%]^{b}$ & 20.7 & 25.5 & 9.5 & 22.6 & 43.7 & 50.8 & 43.8 & 34.9 \\
\hline & $E_{v}[\%]^{b}$ & 1.5 & 1.7 & 1.0 & 1.3 & 1.2 & 1.3 & 1.5 & 2.1 \\
\hline & $E_{v}[\%]^{b}$ & 14.0 & 11.0 & 18.6 & 18.4 & 4.2 & 4.2 & 9.0 & 10.5 \\
\hline & $E_{v}[\%]^{b}$ & 1.5 & 1.7 & 0.8 & 1.2 & 1.6 & 1.6 & 1.8 & 5.4 \\
\hline & $E_{v}[\%]^{b}$ & 37.7 & 39.9 & 30.0 & 43.6 & 50.7 & 58.0 & 56.1 & 52.9 \\
\hline
\end{tabular}

a. horizontal Illuminance at $0.85 \mathrm{~m}$

b. vertical Illuminance in percent of the luxmeter-measured value

origin of light (e.g. by means of light incidence according to $[19,20]$.

Spectral power distribution was not considered in this study. Future research will look into spatially and spectrally resolved measurements, using a luminance camera that includes colorimetric filters and a melanopic filter, to evaluate the consequences of spatial sensitivity on NIF effects of typical lighting conditions with varying spectral power distributions in laboratory studies as well as field studies.

\section{ACKNOWLEDGEMENT}

The authors gratefully acknowledge the contributions of Frederic Rudawski (Technische Univer- sität Berlin) for software development, Alexander Lee (Technische Universität Berlin) for literature research and Tobias Porsch (TechnoTeam Bildverarbeitung $\mathrm{GmbH}$ ) for providing specific data on the luminance camera.

\section{REFERENCES}

1. LiTG (2015), "Über die nicht-visuelle Wirkung des Lichts auf den Menschen," Deutsche Lichttechnische Gesellschaft e.V., Berlin, vol. 1, 2015.

2. C. Schierz, "Zur Photometrie nichtvisueller Lichtwirkungen," in Proc. 2008 Symposium "Licht und Gesundheit", pp. 112-123.

3. P. Teikari, "Spectral modulation of melanopsin responses: role of melanopsin bistability in pupillary 
light reflex," Ph.D. dissertation, Université Claude Bernard-Lyon I, 2012.

4. P. Novotny, P. Paulick, M.J. Schwarz, H. Plischke, "The Solid Angle of Light Sources and Its Impact on the Suppression of Melatonin in Humans," in Proc. $2013 \mathrm{Hu}$ man-Computer Interaction. Towards Intelligent and Implicit Interaction Conf., Lecture Notes in Computer Science, vol 8008. Kurosu, Ed. Berlin, Heidelberg: Springer, 2013, pp. 454-463.

5. J. Y. Wang, J.P. Hanifin, M.D. Rollag and G.C. Brainard, "Ocular regulation of the human pineal gland: The significance of total retinal exposure for melatonin suppression," in: M.F. Holick und E.G. Jung, Ed. Boston, MA: 1998, Biologic Effects of Light 1998: Proceedings of a Symposium Basel, Switzerland November,1998. Springer US, pp. 367-374.

6. G. C. Brainard, M.D. Rollag and J.P. Hanifin, "Photic regulation of melatonin in humans. Ocular and neural signal transduction,” Journal of Biological Rhythms, 1997, Vol. 12, pp. 537-546.

7. E. K. Visser, D. G.M. Beersma, and S. Daan, "Melatonin Suppression by Light in Humans Is Maximal When the Nasal Part of the Retina Is Illuminated” Journal of Biological Rhythms, vol. 14, pp. 116-121.

8. M. Rüger, M. C.M. Gordijn, D. G.M. Beersma, B. de Vries and S. Daan, "Nasal versus Temporal Illumination of the Human Retina: Effects on Core Body Temperature, Melatonin, and Circadian Phase," Journal of Biological Rhythms, 2005, Vol. 20, pp. 60-70.

9. T. A. Lasko, D.F. Kripke and J.A. Elliot, "Melatonin Suppression by Illumination of Upper and Lower Visual Fields,” Journal of Biological Rhythms, vol. 14, pp. 122-125, 1999.

10. J. S. Smith, D.F. Kripke, J.A. Elliot and S.D. Youngstedt, "Illumination of upper and middle visual fields produces equivalent suppression of melatonin in older volunteers,” Chronobiology International, 2002, Vol. 19, pp. 883-891.

11. G. Glickman, J.P. Hanifin, M.D. Rollag, J. Wang, H. Cooper, G.C. Brainard, "Inferior Retinal Light Exposure Is More Effective than Superior Retinal Exposure in
Suppressing Melatonin in Humans," Journal of Biological Rhythms, 2003, Vol. 18, pp. 71-79.

12. H. Piazena, L. Franke, B. Thomsen, I. Kamenzky, R. Uebelhack and S. Völker, "Melatoninsuppression mit Weißlicht-LEDs - erste Ergebnisse," in Proc. 20148. Symposium Licht und Gesundheit, pp. 39-52.

13. C. Schierz, "Ist die Beleuchtungsstärke am Auge die richtige Größe für biologische Lichtwirkungen?" in Proc. 2010 DIN-Expertenforum "Wirkung des Lichts auf den Menschen" pp. 7-17.

14. J. Van Derlofske, A. Bierman, M. S., Rea and N. Maliyagoda, "Design and optimization of a retinal exposure detector", in Proc. 2000 International Society for Optics and Photonics - International Symposium on Optical Science and Technology, pp. 60-70.

15. J. Van Derlofske, A. Bierman, M. S., Rea, J. Ramanath and J.D. Bullough, "Design and optimization of a retinal flux density meter," Measurement Science and Technology, 13(6), 821, 2002.

16. FGL (Fördergemeinschaft Gutes Licht) (2014), "licht.wissen 19 Impact of Light on Human Beings," [Online]. Available: http://en.licht.de/fileadmin/shop-downloads/1409_LW19_E_Impact-of-Light-on-Human-Beings_web.pdf.

17. M. Rüger, M. C.M. Gordijn, D. G.M. Beersma, B. de Vries and S. Daan, "Weak relationships between suppression of melatonin and suppression of sleepiness/ fatigue in response to light exposure," Journal of Sleep Research, 2005, Vol. 14, pp. 221-227.

18. R. J. Lucas, S.N. Peirson, D.M. Berson, T.M. Brown, H.M. Cooper, C.A. Czeisler et al., "Measuring and using light in the melanopsin age," Trends in neurosciences, 2014, Vol. 37, pp. 1-9.

19. C. Liedtke, S. Völker and M. Knoop, "The Light Direction and Directional Light - Towards a new Quantification of an Essential Lighting Quality Criterion," in Proc. 2013 CIE Centenary Conf., pp.542-551.

20. M. Knoop, A. Diakite, C. Liedtke, K. Broszio, M. Niedling, "Characterisation of daylight's spatial and spectral distribution to assess its impact on human beings, “ unpublished, presented at CIE Midterm Meeting, Jeju Island, Republic of Korea, 2017.

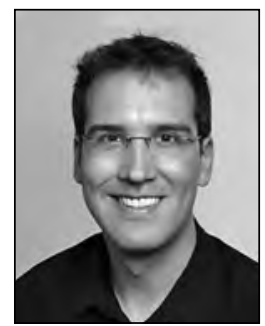

Kai Broszio is a Ph.D. student in the field of non-image forming effects on humans at the Chair of Lighting Technology, TU Berlin. He studied Electrical Engineering with a specialization on lighting technology and photovoltaic and worked on the European project Energy Saving Outdoor Lighting (ESOLi). He is currently working as teaching and research assistant at the Chair of Lighting Technology. His doctoral thesis deals with the impact of directionality of light on non-image forming effects. He is a guest member of the indoor lighting expert group of the German Society of Lighting Technology (LiTG) 


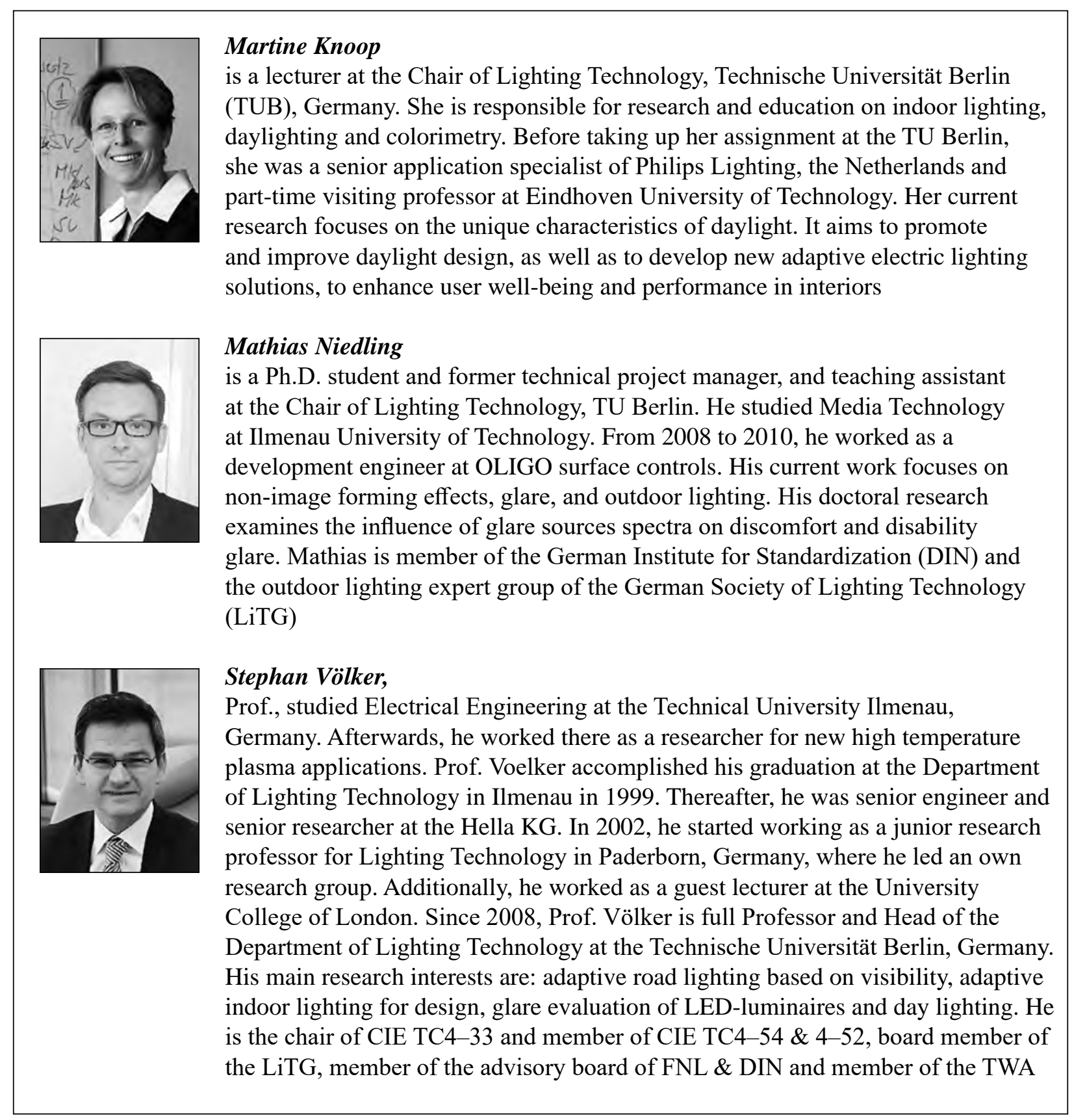

